Nonlin. Processes Geophys. Discuss., doi:10.5194/npg-2016-11, 2016

Manuscript under review for journal Nonlin. Processes Geophys.

Published: 1 February 2016

(c) Author(s) 2016. CC-BY 3.0 License.

\title{
Linear and Non-linear Stability Analysis of the Rate and State Friction Model with Three State Variables
}

\author{
Nitish Sinha, Arun K. Singh \\ Visvesvaraya National Institute of Technology, Nagpur-440010, INDIA \\ nitishme08@gmail.com_aksinghb@gmail.com
}

\begin{abstract}
In this article, we study linear and non-linear stability of the three state variables rate and state friction $(3 \mathrm{sRS})$ model with spring-mass sliding system. Linear stability analysis shows that critical stiffness, at which dynamical behaviour of the sliding system changes, increases with number of state variables. The bifurcation diagram reveals that route of chaos is period doubling and this has also been confirmed with the Poincaré maps. The present system is hyperchaos since all Lyapunov exponents are positive. It is also established that the 3sRSF model is more chaotic than corresponding to the 2sRSF model. Finally, the implication of the present study is also discussed.

\section{Introduction}

One of the most important applications of friction in recent decades is in understanding the sliding dynamics of earthquake faults (Brace and Byerlee, 1966; Dieterich, 1979; Rice and Ruina, 1983). It is believed that the stick-slip process along the earthquake faults results in earthquakes. Researchers use rate and state friction(RSF) model oftenly to explain the earthquake process (Brace and Byerlee, 1966; Dieterich, 1979; Rice and Ruina, 1983). The RSF model was proposed by Dieterich (1979,1981), Ruina (1983) and Ruina and Rice (1983). Although the RSF model is an empirical model, its genesis has been explained using the Eyering's rate reaction theory (Rice et. al., 2001). Classical Amontons-Coulombs' (AC) laws are widely used for explaining variety of friction based phenomena of hard solids (Persson,2000). Nonetheless these friction laws do not explain many observations for instance increase in friction with time of contact and sliding velocity, more significantly, stiffness dependence of stick-slip behavior etc. (Rice and Ruina,1983). In fact, these 
Nonlin. Processes Geophys. Discuss., doi:10.5194/npg-2016-11, 2016

Manuscript under review for journal Nonlin. Processes Geophys.

Published: 1 February 2016

(c) Author(s) 2016. CC-BY 3.0 License.

1 limitations of the AC laws led to the proposal of the modified friction model which is known

2 as the rate and state friction (RSF) model. According to this friction model of hard solids such

3 as rock solids depends on the "slip rate" as well as the "state" of the sliding surfaces (Rice and

4 Ruina,1983; Ruina,1983). Although one state variable explains well the stiffness dependence

5 of stick-slip oscillatory motion of a sliding mass, it doesn't explain its chaotic behavior. As a

6 result, one state variable RSF law has been modified by introducing an additional state

7 variable by believeing that chaos is a manifestation of more complex friction processes at the

8 slip interface. This observation led to the proposal of the two state variables rate and state

9 dependent friction (2sRSF) model. The 2sRSF model shows the chaotic behavior ( Ruina, 1983; Gu et. al., 1984; Gu and Wong, 1994; Zhiern and Dangmin, 1994; Niu and Chen, 1995;

11 Becker, 2000; Gao, 2013). It arises naturally a question what happens to the 2 sRSF model if one more state variable is added in this friction model. In this article we have studied numerically linear and nonlinear dynamics of the three state variables rate and state friction(3sRSF) with spring-mass sliding system. The results are also compared with the corresponding two state variables rate and state friction (2sRSF) model.

Chaos is defined as "Aperiodic long-term behavior in a deterministic system that exhibits sensitive dependence on initial conditions" (Strogatz,1994). The conditions for a continuous dynamical system to be chaotic are that the governing differential equation must possess at least three independent variables, and also show the dependence on initial conditions (Devany,1989). There are many well known and extensively studied chaotic systems in literature for example Duffing oscillator, Lorenz system, Rössler system etc. (Strogatz,1994). Moreover, phase plot, Poincaré maps, bifurcation diagram, Lyapunov exponents etc. are the numerical tools which are widely used for studying chaotic behavior of a dynamical system. Rössler introduced the concept of hyperchaos by modifying one of the simplest chaotic models (Rössler,1979). The general conditions for the hyper-chaos are that the system of 
Nonlin. Processes Geophys. Discuss., doi:10.5194/npg-2016-11, 2016

Manuscript under review for journal Nonlin. Processes Geophys.

Published: 1 February 2016

(c) Author(s) 2016. CC-BY 3.0 License.

1 differential equations should have at least four independent variables and the system must

2 also be dissipative (Wang and Wang,2008; Chen et. al., 2006). Moreover, the Lyapunov

3 exponents of the dynamical system must show at least two positive, one zero and one negative

4 (Niu and Chen,1995). Further, the sum of all Lyapunov exponents must be negative

5 (Moghtadaei and Goplaegani,2012). In additions to these conditions, the phase plot should

6 also show twisting structure in the chaotic behavior (Moghtadaei and Goplaegani, 2012).

7 Notwithstanding the aforementioned conditions for hyperchaos, there are dynamical systems

8 which have been claimed to be hyper chaos. For example, Oteski et al. (2015) have claimed

9 that an air-filled differentially heated cavity to be hyperchaotic on the basis of all positive Lyapunov exponents(LEs). In the present 3sRSF model as well, we will establish numerically that all LEs are positive hence the 3sRSF dynamical system to be hyperchaotic. In literature majority of study has been done with one state variable based RSF law (Ranjith and Rice, 1999). The reason may be attributed to the fact that one state variable based friction law is enough to explain the stick-slip phenomenon or frictional instability of hard surfaces. Gu et al. (1984) have studied numerically the linear and non-linear behaviour of the springmass slider with the $1 \mathrm{sRSF}$ model as well as the $2 \mathrm{sRSF}$ model. They have reported stick-slip behavior with $1 \mathrm{sRSF}$ model while the 2 sRSF model shows the period doubling as well as chaotic behaviour. $\mathrm{Gu}$ and Wong (1992) have carried out linear and nonlinear stability analysis with both the $1 \mathrm{sRSF}$ and $2 \mathrm{sRSF}$ models using the tools phase portraits, time series,

20

21 and bifurcation diagrams. They have established that the most significant parameter is spring stiffness which controls the stability of the sliding mass. Zhiren and Dangmin $(1994,1995)$ have carried out the numerical simulations of $2 \mathrm{sRSF}$ model with the slip law, and they observed that the sliding system shows the quasi-periodic to chaotic behaviour upon decrease in spring stiffness even in the absence of inertia that is, under the quasistatic conditions. They have also estimated the Lyapunov exponents as well as Lyapunov dimensions to confirm the 
Nonlin. Processes Geophys. Discuss., doi:10.5194/npg-2016-11, 2016

Manuscript under review for journal Nonlin. Processes Geophys.

Published: 1 February 2016

(c) Author(s) 2016. CC-BY 3.0 License.

1 evidence of chaotic behaviour of the system (Niu and Chen, 1995). Xuejun(2013) has

2 investigated the stability of the $2 \mathrm{sRSF}$ and finds the period doubling route to chaos. Wang

3 (2002,2009) has pointed out that the "slip" and "slowness" laws differ in high velocity

4 regime but not in the low velocity sliding regime. In recent times the 2 sRSF model has been

5 used to validate the experimental data concerning rock friction at high temperature in the

6 framework of the 2sRSF(Liu, 2007 King and Marone,2012). Nontheless these researchers

7 have not reported any evidence of chaotic behavior in the experiments at high temperature.

8 The present analysis is related with the three state variable RSF model i.e., the 3sRSF model.

9 The organization of the paper is as following. First we have derived governing differential equations of the spring-mass sliding system with $3 \mathrm{sRSF}$ in non-dimensional form following

11 the same procedure as was done by Xuejun[2013]. It is then linear stability of Eq. (4) is

carried out by linearizing about steady state or equilibrium points. The expression for critical stiffness is also derived using Routh- Hurwitz criterion (Persson,2000). The physical meaning of the critical stiffness is that at this value of stiffness the sliding behavior changes from unstable to stable sliding or vice versa. The non-linear analysis of Eq. (4) is also carried out in detail with different tools such as phase plot, Poincaré maps, bifurcation diagram, Lyapunov exponents and Lyapunov dimensions. Finally a comparative study is also done between 2 sRSF and 3sRSF models to justify the present results.

\section{Modelling of Spring-mass system with three state variables friction law}

According to the rate and state friction(RSF) model, frictional stress ${ }^{6} \tau$ ' of a sliding hard surface depends on sliding velocity ' $v$ ' and state variable ' $\theta$, (Ruina, 1983). Based on the experimental observations Dieterich(1978), Ruina(1980,1983), Ruina and Rice(1983) proposed the following empirical relation

$$
\tau=\tau^{*}+\theta_{i}+A \ln \frac{v}{v^{*}} \text {, and } \frac{d \theta_{i}}{d t}=-\frac{v}{L_{i}}\left[\theta_{i}+B_{i} \ln \frac{v}{v^{*}}\right] .
$$


Nonlin. Processes Geophys. Discuss., doi:10.5194/npg-2016-11, 2016

Manuscript under review for journal Nonlin. Processes Geophys.

Published: 1 February 2016

(c) Author(s) 2016. CC-BY 3.0 License.

1 where $\theta_{i}$ is number $(i=1,2,3 \ldots)$ of state variables, $B_{i}$ are $L_{i}$ are the constants. Further ' $\tau^{*}$, and

2 ' $v$ "' are reference frictional shear stress and shear velocity respectively. The system of

3 differential equations in Eq.(1) with three state variables are expanded as

4

$$
\begin{aligned}
& \tau=\tau^{*}+\theta_{1}+\theta_{2}+\theta_{3}+A \ln \frac{v}{v^{*}}, \& \frac{d \theta_{1}}{d t}=-\frac{v}{L_{1}}\left[\theta_{1}+B_{1} \ln \frac{v}{v^{*}}\right] . \\
& \frac{d \theta_{2}}{d t}=-\frac{v}{L_{2}}\left[\theta_{2}+B_{2} \ln \frac{v}{v^{*}}\right], \& \frac{d \theta_{3}}{d t}=-\frac{v}{L_{3}}\left[\theta_{3}+B_{3} \ln \frac{v}{v^{*}}\right] .
\end{aligned}
$$

5 Dieterich(1979), Ruina(1983) have proposed two laws governing the "state" of the sliding

6 surfaces which are know as the Ruina-Rice slip law or simply slip law and Dieterich-Ruina

7 ageing law or ageing law [3]. It is important to note that the, unlike ageing law, the slip law of

8 the RSF model shows chaotic behaviour (King and Marone,2012 ). The reason for this

9 contradictory observation is not yet reported in literature.

10 In order to study the 3sRSF model, we have also used the spring-mass sliding system under

11 the quasi-static conditions. The free end of the spring having spring constant $k\left(\operatorname{Pam}^{-1}\right)$ is

12 being pulled constantly with a constant pulling velocity ' $v_{0}$ ' as a result the rate of change of

13 friction at the sliding interface is given by

14

$$
\frac{d \tau}{d t}=k\left(v_{0}-v\right)
$$

The non-dimension form of above set of Eqs.(1-5) are expressed by introducing non-

16 dimension variables as velocity $\phi$, shear stress $f$, state variables $\hat{\theta}_{1}$, and $\hat{\theta}_{2}$, time T, pulling

17 velocity $\phi_{0}$,spring stiffness $\mathrm{K}$

18

$f=\frac{\tau-\tau^{*}}{A}, \phi=\ln \frac{v}{v^{*}}, \quad \hat{\theta}_{1}=\frac{\theta_{1}}{A}, \quad \hat{\theta}_{2}=\frac{\theta_{2}}{A}, \quad \mathrm{~T}=\frac{\mathrm{v}^{*}}{\mathrm{~L}_{1}} t, \beta_{1}=\frac{B_{1}}{A}, \beta_{2}=\frac{B_{2}}{A}, \beta_{3}=\frac{B_{3}}{A}, \rho=\frac{\mathrm{L}_{1}}{\mathrm{~L}_{2}}$,

$$
\rho_{1}=\frac{\mathrm{L}_{1}}{\mathrm{~L}_{3}}, \quad \phi_{0}=\ln \frac{v^{0}}{v^{*}}, \mathrm{~K}=k \frac{L_{1}}{A} \text {. }
$$


Nonlin. Processes Geophys. Discuss., doi:10.5194/npg-2016-11, 2016

Manuscript under review for journal Nonlin. Processes Geophys.

Published: 1 February 2016

(c) Author(s) 2016. CC-BY 3.0 License.

1 Non-dimensional form of the system of differential equations Eq.(4) is obtained using Eqs. (2-

2 3). After having eliminated the third state variable $\hat{\theta}_{3}$, we get the following system of

3 differential equations:

4

$$
\left\{\begin{array}{l}
\frac{d \phi}{d T}=e^{\phi}\left[\left(1-\rho_{1}\right) \hat{\theta}_{1}+\left(\rho-\rho_{1}\right) \hat{\theta}_{2}+\left(\beta_{1}+\rho \beta_{2}+\rho_{1} \beta_{3}-\rho\right) \phi+\rho_{1} f-K\right]+K e^{\phi_{0}} \\
\frac{d f}{d T}=K\left(e^{\phi_{0}}-e^{\phi}\right) \\
\frac{d \hat{\theta}_{1}}{d T}=-e^{\phi}\left(\hat{\theta}_{1}+\beta_{1} \phi\right) \\
\frac{d \hat{\theta}_{2}}{d T}=-\rho e^{\phi}\left(\hat{\theta}_{2}+\beta_{2} \phi\right)
\end{array}\right.
$$

5 It may be noted that Eq.(4) is having four variables i.e, four dimensional system, thus we

6 investigate the possibility of hyperchaos in Eq.(4).

$7 \quad 3.0$. Results and Discussion

\section{$8 \quad$ 3.1.Linear Stability analysis}

9 Linear stability of the spring-mass model is done about steady state or equilibrium point(Strogratz, 1994). The equilibrium or fixed points are obtained by equating the equations to zero. The equilibrium points of Eq.(4) are obtained as

$$
\phi_{s s}=\phi_{0}, \theta_{1 s s}=-\beta_{1} \phi_{0}, \hat{\theta}_{2 s s}=-\beta_{2} \phi \text {, and } f_{s s}=\left(\frac{\rho}{\rho_{1}}-\beta_{1}-\beta_{2}-\beta_{3}\right) \phi_{0}
$$

The charactersticequation $\left|J_{0}-\lambda I\right|=0$, is expanded for polynomial equation in terms of eigen value $\lambda$. where $J_{0}$ is Jacobian matrix of Eq.(4) about the steady state and $I$ is identity matrix. Routh-Hurwitz criterion is used to obtain critical stiffness $k_{c r}$ at which sliding behaviour of the spring-mass system changes. Other details about evaluating $k_{c r}$ is given in appendix-I.

17 The physical significance of $k_{c r}$ is that the sliding system changes its behaviour from unstable

18 to stable sliding for spring stiffness larger than $k_{c r}$ (Gu et. al., 1984; Ranjith and Rice,1999).

19 For instance, Fig.1 presents the results that the sliding system is dynamically unstable for 
Nonlin. Processes Geophys. Discuss., doi:10.5194/npg-2016-11, 2016

Manuscript under review for journal Nonlin. Processes Geophys.

Published: 1 February 2016

(c) Author(s) 2016. CC-BY 3.0 License.

stiffness $k=0.2633$ and neutral for critical stiffness $k_{c r}=0.2635$ and stable for stiffness the 1s RSF model (Ranjith and Rice,1999).

6 Fig.1. Stiffness dependent sliding behavior of spring-mass for $\beta_{1}=1.2, \beta_{2}=0.84, \beta_{3}=0.38$, 7 8

\section{9}

10

11

12

13

14

15

16

17

18

$\rho=0.048$ and $\rho_{1}=0.034$ for initial condition $[0.19885,-1.00824,-0.23862,-0.167034]$.

\subsection{Effect of friction parameters on critical stiffness}

The effect of friction parameter such as $\beta_{1}$ is investigated on critical stiffness $k_{c r}$ numerically.

The values of friction parameters are considerd the same as in literature[Rice and Ruina,1983;

Gu. et. al.,1984]. However numerical values of additional parameters $\beta_{3}$ and $\rho_{1}$ in the 3sRSF model are estimated on the basis of the reported values in literature (Gu. et. al., 1984) For instance, friction parameter $\beta$ decreases if friction law is modified from one state variable to two state variables. The result in Fig.2 shows that $k_{c r}$ increases linearly with $\beta_{1}$. This linear behaviour is also seen with the $2 \mathrm{sRSF}$ law though we are not presenting the results here. The dependence of critical stiffness in the 3sRSF model with respect to variables, for instance $\beta_{2}, \beta_{3}, \rho$ and $\rho_{1}$, is also linear though we have not presented the results here.
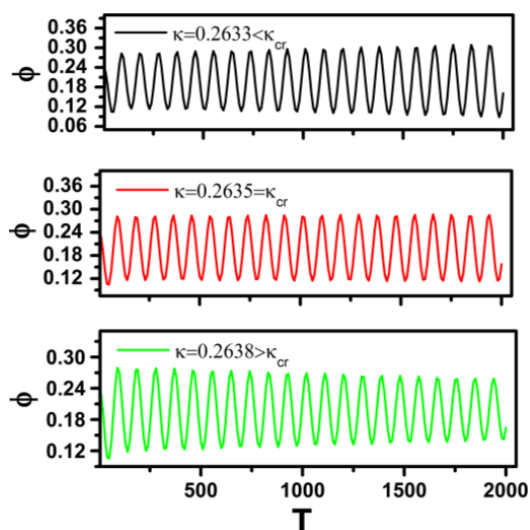
Nonlin. Processes Geophys. Discuss., doi:10.5194/npg-2016-11, 2016

Manuscript under review for journal Nonlin. Processes Geophys.

Published: 1 February 2016

(c) Author(s) 2016. CC-BY 3.0 License.

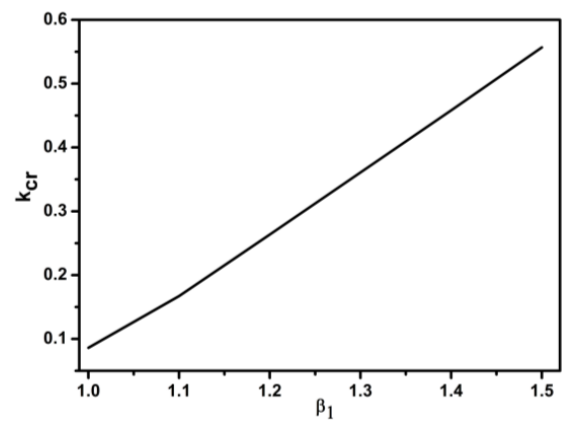

2 Fig.2. Effect of friction parameter $\beta_{1}$ on critical stiffness $k_{c r}$ for $\beta_{2}=0.84, \beta_{3}=0.38$, $3 \rho=0.048$ and $\rho_{1}=0.034$.

4

5

6

\subsection{Nonlinear stability analysis}

Motivated from linear stability analysis, we have also carried out non-linear stability of the system of governing differential equations in Eq.(4). This is solved with MATLAB ${ }^{\circledR}$ using ode23s solver for ordinary differential equations. Fig.3 shows the single orbit in phase portrait, which means the system behaviour is periodic at spring stiffness $k=0.087$. This has also been confirmed using Poincaré section which shows single point in the map in Fig.3.
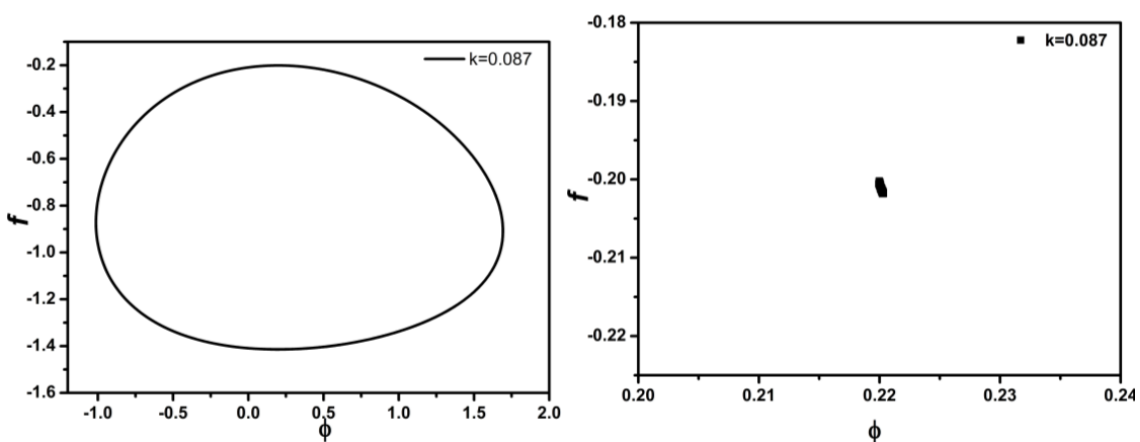

Fig.3 phase diagram(left) $f$ vs. $\phi$ and Poincaré section(right) for $k=0.087, \beta_{1}=1.0$, $\beta_{2}=0.84, \beta_{3}=0.38, \rho=0.048$ and $\rho_{1}=0.034$ for initial condition $[0,0,0,0]$.

Now upon lowering the magnitude of spring stiffness to $k=0.085$, Fig.4 shows the evidence of period doubling and this phenomena is also confirm by the Poincaré map. As Poincaré section in Fig.4 shows two points. 
Nonlin. Processes Geophys. Discuss., doi:10.5194/npg-2016-11, 2016

Manuscript under review for journal Nonlin. Processes Geophys.

Published: 1 February 2016

(c) Author(s) 2016. CC-BY 3.0 License.
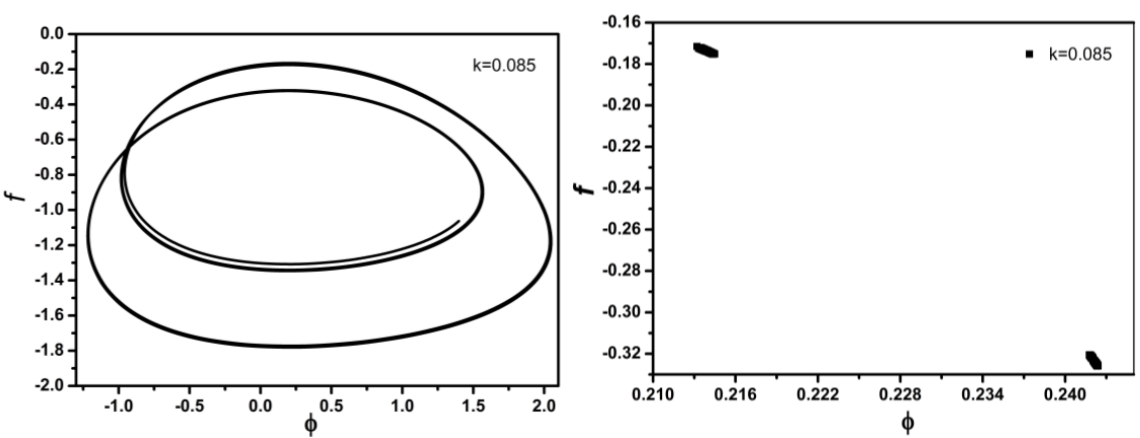

Fig.4 phase diagram $f$ vs. $\phi$ and corresponding Poincaré section for $k=0.085, \beta_{1}=1.0$, $\beta_{2}=0.84, \beta_{3}=0.38, \rho=0.048$ and $\rho_{1}=0.034$ for initial condition $[0,0,0,0]$.

As magnitude of stiffness decreases further to $k=0.08437$, the dynamical behaviour of the system changes further. Now the phase portrait in Fig.5 results in period quadrupling and this is also confirmed in the corresponding Poincaré section in Fig.5.
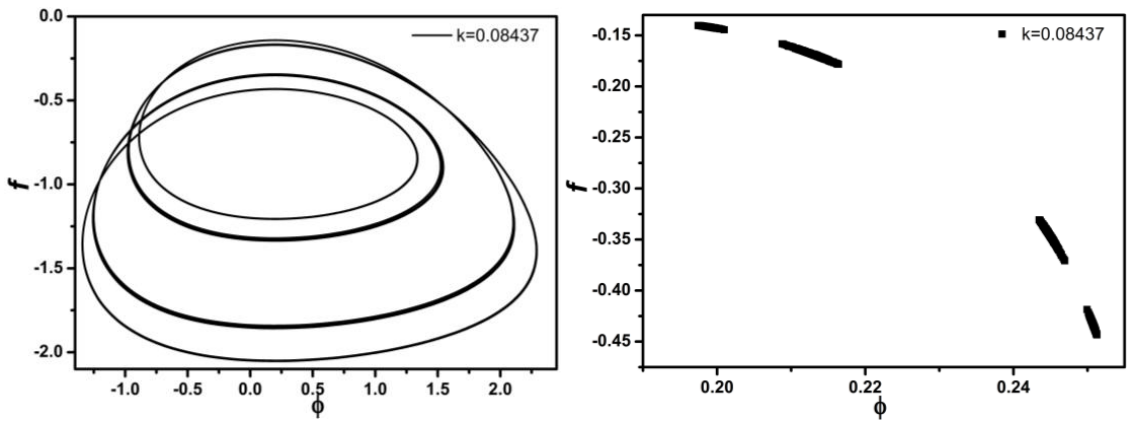

Fig.5 phase diagram $f$ vs. $\phi$ and corresponding Poincaré section for $k=0.08437, \beta_{1}=1.0$, $\beta_{2}=0.84, \beta_{3}=0.38, \rho=0.048$ and $\rho_{1}=0.034$ for initial condition $[0,0,0,0]$.

As the controlling parameter $k$ decreases further, the 3sRSF leads the spring-mass system in chaos. For instance,Fig.6 presents the phase diagram and corresponding Poincaré section for $k=0.08421$. The phase portrait shows infinite period with bounded orbits and the corresponding Poincaré section in the form of continuous line. 
Nonlin. Processes Geophys. Discuss., doi:10.5194/npg-2016-11, 2016

Manuscript under review for journal Nonlin. Processes Geophys.

Published: 1 February 2016

(c) Author(s) 2016. CC-BY 3.0 License.
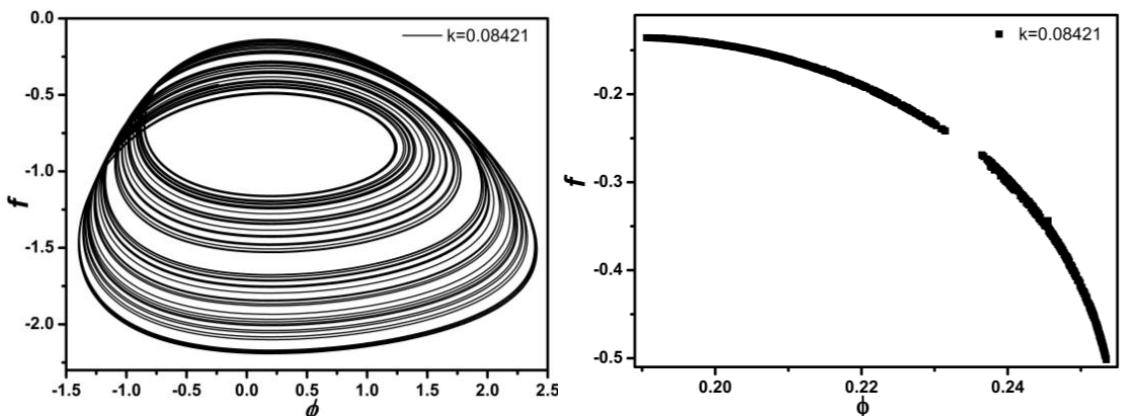

Fig.6 phase diagram $f$ vs. $\phi$ and corresponding Poincaré section for $k=0.08421, \beta_{1}=1.0$, $\beta_{2}=0.84, \beta_{3}=0.38, \rho=0.048$ and $\rho_{1}=0.034$ for initial condition $[0,0,0,0]$.

A physical significance of the present results is in nucleation of earthquake process. For instance, The phase portraits in Fig.3-6 show an interesting observation that frictional stress as well as corresponding slip velocity at the sliding interface changes from periodic to chaotic upon decreasing spring stiffness of the slider. This results in a direct surge of stress amplitude thus the nucleation of earthquake process occurs. This observation is similar to the chaotic nature of the sliding mass with the 2sRSF in which magnitude of the stress fluctuates considerably thus the earthquake nucleation begins (Becker, 2000).

\subsection{Bifurcation diagram}

The results in Figs.(3-6) have also been confirmed by the bifurcation diagram in shown Fig.7. In the bifurcation diagram the control parameter in the form of non-dimensional stiffness $k$ decreases by a small step $10^{-6}$ from $k=0.089$ to $k=0.084$, the evolution of the system is initially periodic oscillation with increasing amplitude as evident in Fig.7. Upon further decrease in stiffness upto $k=0.085$, the behaviour of the system changes to period doubling as obvious in Fig.7. If stiffness decreases to further lower value i.e., $k=0.08437$, the system behaviour bifurcates to the period four (Fig.7). Finally the system results in chaotic behaviour at minimum stiffness $k=0.08421$. These results are in confirmation with phase portraits and Poincaré section in Figs.(3-6). 
Nonlin. Processes Geophys. Discuss., doi:10.5194/npg-2016-11, 2016

Manuscript under review for journal Nonlin. Processes Geophys.

Published: 1 February 2016

(c) Author(s) 2016. CC-BY 3.0 License.

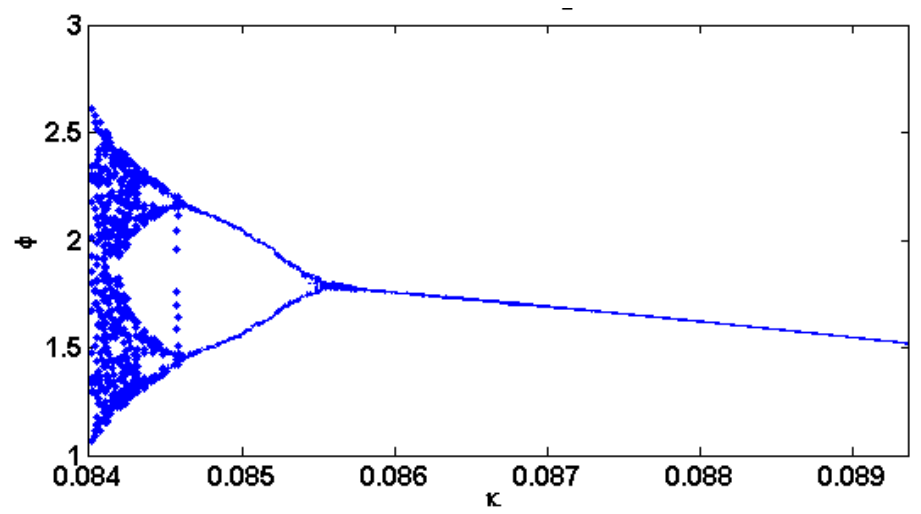

Fig.7 Bifurcation diagram for spring stiffness $k=0.87$ to $0.084, \beta_{1}=1.0, \beta_{2}=0.84$, $\beta_{3}=0.38, \rho=0.048$ and $\rho_{1}=0.034$ for initial condition $[0,0,0,0]$.

Fig.7 also summarizes the variation of velocity amplitude with decreasing spring stiffness. It is obvious that the overall velocity amplitude increase from periodic to chaotic way as stiffness of the connecting spring decreases. This observation is consistent with the phase plots in Figs.3-6.

\subsection{Lyapunov exponent and dimensions}

Lyapunov exponent(LE) is the most significant tool for investigating the dynamical behavior of a physical system (Kaplan and Yorke,1979). We have used the MATLAB ${ }^{\circledR}$ program for evaluating the LE of the present dynamical system by Lyapunov Exponent Toolbox (LET), which is developed by Steve SIU (1998). For the four-dimensional dissipative system, there are three possible type of strange attractors such as the combination of Lyapunov spectra as $(+,+, 0,-),(+, 0,0,-)$ and $(+, 0,-,-)$ (Wolf. et. al., 1985). If LE is negative the dynamical system is stable with dissipative in nature, while the positive LE signifies the system become unstable orbit or chaotic. However, LE with zero magnitude signifies the system is dynamically neutral (Wolf. et. al., 1985).

The present analysis of the 3sRSF model shows in Fig.8 that the magnitude of LEs are $\mathrm{LE}_{1}=1.8146, \mathrm{LE}_{2}=0.0461, \mathrm{LE}_{3}=0.0577, \mathrm{LE}_{4}=0.0351$. This result confirms that the present dynamical system is very similar to a hyperchao as more than one Lyapunov exponents is 
Nonlin. Processes Geophys. Discuss., doi:10.5194/npg-2016-11, 2016

Manuscript under review for journal Nonlin. Processes Geophys.

Published: 1 February 2016

(c) Author(s) 2016. CC-BY 3.0 License.

1 positive (Oteski. et. al., 2015). At the same time, the magnitude of three LEs are one order

2 less than the remaining one. This result is in contrast with the 2 sRSF in which results are one

3 positive, one negative and one zero in magnitude (Niu and Chen,1995). The relationship

4 between the Lyapunov exponents and fractal dimensions is established by Kaplan and

5 Yorke(1979). They have proposed the Lyapunov or Kaplan-Yorke dimension $D_{K Y}$ which is

6 given by the formula: $D_{K Y}=D+\frac{1}{\left|h_{D}+1\right|} \sum_{i=1}^{D} h_{i}$

7 where $D$ is the largest integer for which $\sum_{i=1}^{D} h_{i}>0$. As a result, $D_{K Y}$ is a convenient

8 geometrical measure of objects in phase space if Lyapunov exponents are known. The fractal

9 dimension of the present dynamical system is calculated to be as 5.70 .

10

11 Fig.8.Lypunov exponents vs. .time for $\mathrm{k}=0.08421, \beta_{1}=1.0, \beta_{2}=0.84, \beta_{3}=0.38, \rho=0.048$

12

13

14

15

16

17

and $\rho_{1}=0.034$ for initial conditions $[0,0,0,0]$

The 3 sRSF based quasistatic system also follows the universal period doubling route to chaos.

The Feigenbaum number is estimated using given formula $\delta_{n}=\left(k_{n+1}-k_{n}\right) /\left(k_{n+2}-k_{n+1}\right)$ where $n=1,2,3 \ldots$, this number should converge to Feigenbaum number 4.669201. we have calculated Feigenbaum universality constant for 3sRSF law and estimated to 3.9375. However

this single value does not indicate the sign of convergence. It may be possible that

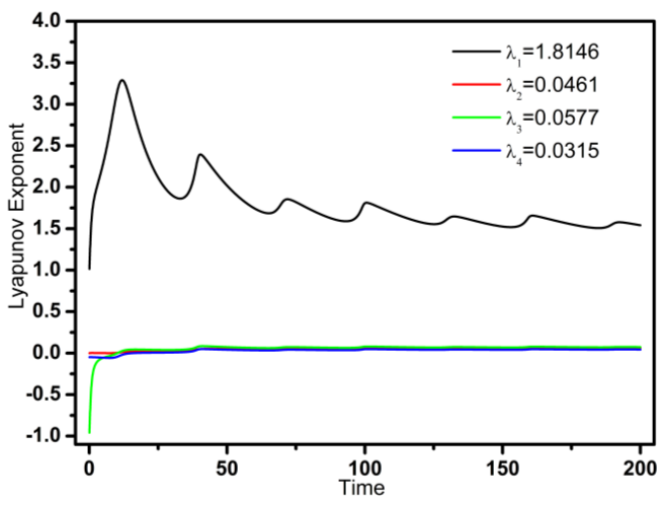


Nonlin. Processes Geophys. Discuss., doi:10.5194/npg-2016-11, 2016

Manuscript under review for journal Nonlin. Processes Geophys.

Published: 1 February 2016

(c) Author(s) 2016. CC-BY 3.0 License.

convergence for the bifurcation sequence to chaos for the friction model is different from the

2 logistic map.

3

4 We have also investigated whether the present friction model fulfils the other conditions of hyperchaos. The hyperchaotic behavior in the form of phase portraits in Fig.9 shows the twisting nature of the phase diagram. This is also a feature of hyper chaos().
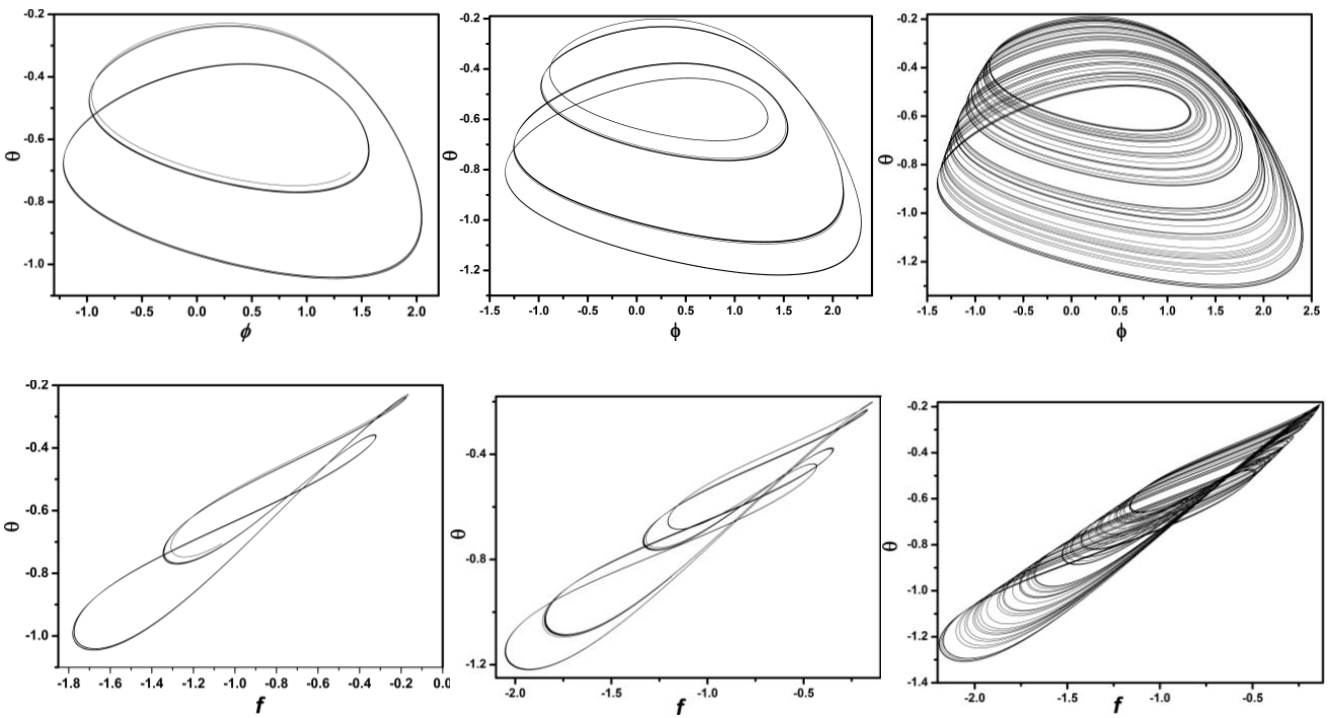

Fig.9. Twisted phase diagram for stiffness value (a) $k=0.085$, (b) $k=0.08437$, (c) $k=0.08421$ and $\beta_{1}=1.0, \beta_{2}=0.84, \beta_{3}=0.38, \rho=0.048$ and $\rho_{1}=0.034$ for initial condition $[0,0,0,0]$.

12

We have also compared the linear and non-linear behavior between the 2 sRSF and the $3 \mathrm{sRSF}$ models. For instance, critical stiffness, at which dynamics of stick-slip motion changes, increases with number of state variables. Moreover, the route of chaos is same for both 2sRSF and 3sRSF models, that is, period doubling. But period eight and period sixteen are not observed in the present system which is unlike to the 2sRSF model (Xuejun,2013). Moreover,LEs of the $2 \mathrm{sRSF}$ are reported to be one positive, one negative and one zero. The 3sRSF model, in contrast, shows all four LEs are positive. This result has been confirmed 
Nonlin. Processes Geophys. Discuss., doi:10.5194/npg-2016-11, 2016

Manuscript under review for journal Nonlin. Processes Geophys.

Published: 1 February 2016

(c) Author(s) 2016. CC-BY 3.0 License.

1 using magnitude of fractal dimension (FD). For example, FD of the 2sRSF is 2.11 which is

2 less than the FD of 3sRSF i.e., 5.7. Moreover, Poincaré section of the 3 sRSF model is

3 slightly intricate than the $2 \mathrm{sRSF}$ model. On the basis of these evidances, it is established that

4 the 3 sRSF model is more chaotic than the 2 sRSF model.

5 5.Conclusions

6 We have established numerically that the three state variables based RSF model show the chaotic behavior. All Lyapunov exponent is positive. The route of chaos is established to be period doubling bifurcation. Moreover, critical stiffness of the dynamical system increases with number of state variables. It is also observed that the $3 \mathrm{sRSF}$ is more chaotic than corresponding to the $2 \mathrm{sRSF}$. It is shown that the $3 \mathrm{sRSF}$ model is hyperchaotic as it exhibits all positive Lyapunov exponents.

12 References:

13 Brace,W.F.,Byerlee,J.D.:Stick-slip as a Mechanism of earthquake,Science,153,990-992,1966

14 Chen,A.,Lu,J.,Lu,J.,Yu,S..:GeneratinghyperchaoticLü attractor via state feedback control,Physica A.

$15364,103-110,2006$

16 Dieterich, J.D.:Modeling of rock friction:1.experimental results and constitutive equation, J.

17 Geophys.Res., 84(B5), 2161-2168,1979

18 Devany.R.:An introduction to chaotic dynamical system, est view press, 1989

19 Feigenbaum,M.J.:Universal behavior in Nonlinear System. Physica.7D,16,1983

$20 \mathrm{Gu}, \mathrm{Y}$. and Wong, T.-F.:Nonlinear dynamics of the transition from stable sliding to cyclic stick-slip in

21 rock, in Nonlinear dynamics and predictability of geophysicalphenomena, vol. 8:3,

22 GeophysicalMonograph, edited by W.I.Newman, A. Gabriclov, and D. L. Turcottc, pp. 15-35,1994,

23 AGU, Washington, D.C.

24 Gu J.C., Rice,J.R., Ruina, A.L. and Tse, S.T.: Slip motion and stability of a single degree of freedom elastic system with rate and state dependent friction. J. Mech. Phys. Solids.32, 167-196,1984

26 Jeen-HwaWang.:A Dynamic Study of Two One-State-Variable, Rate-Dependent, and State-

27 Dependent Friction Laws, Bulletin of the Seismological Society of America. 92,687-694,2002

28 Jeen-HwaWang.:A Numerical study of comparision of two one-state-variable, rate-and-state- 
Nonlin. Processes Geophys. Discuss., doi:10.5194/npg-2016-11, 2016

Manuscript under review for journal Nonlin. Processes Geophys.

Published: 1 February 2016

(c) Author(s) 2016. CC-BY 3.0 License.

King,D.S.H.,Marone,C.:Frictional properties of olivine at high temperature with applications to the strength and dynamics of the oceanic lithosphere. J. Geophys. Res.117, B12203,2012

Wolf, A., Jack, B.S., Harry, L. S., John, A. V.:Determineing Lyapunov exponents from a time series. Physica, 16D, 285-317,1985

Kaplan,J.L.,Yorke,J.A.:Chaotic behavior of multidimensional difference equation, in Functional Differential Equation and Approximation of Fixed Points, Vol. 730,Lecture Notes in Mathematics, edited by H.-O. Peitgen and H.-O. Walter, pp. 204-227,1979 Springer,Berlin

Moghtadaei,M.,Goplayegani,M.R.H.:Complex dynamics behaviours of the complex Lorenz system, ScientiaIranica D.19(3),733-738,2012 Niu, Z.-B. and Chen, D.-M.:Lyapunov exponent and dimension of the strange attractor of elastic frictional system,ActaSeimol. Sinica.8,575-584,1995 O.E. Rossler.:An equation for Hyperchaos, Physics Letters.71A,2-3,1979 Oteski, L., Daguet, Y., Pastur, L., and Quèrè, P.L.:Quasiperiodic routes to chaos in confined twodimensional differential convection., Phys.Rev. E ,92 043020,1-15,2015 Persson Bo. N. J.:Sliding friction physical principal and application, $2^{\text {nd }}$ ed., springer. Verlag Berlin Heidelberg New York,2000

Rice, J.R., Ruina, A.L.:Stability of steady frictional slipping.J.Appl.Mech.50, 343-349,1983

Ruina A.L.:slip instability and state variable friction laws.B12,88,10359-10370,1983

Rice,J.R, Lapusta,N.,Ranjith,K.: Rate and state dependent friction and the stability of sliding between elastically deformable solids. Journal of the Mechanics and Physics of Solids, 491865 - 1898,2001

Ranjith,K.,Rice,J.R.:Stability of quasi-static slip in a single degree of freedom elastic system with rate and state dependent friction"Journal of the Mechanics and Physics of Solids. 47, 12071218,1999

Roy, M.,Marone,C.:Earthquake nucleation on models faults with rate and state dependent friction: The effects of inertia, J. Geophys. Res.101,13919 - 13932,1996

Steven H. Strogatz.: Nonlinear dynamics and chaos,Perseus books publishing:Cambridge,MA,1994

Thorsten W. Becker.:Deterministic Chaos in two state-variable friction slider and effect of elastic itteraction,Geocomplexity and the Physics of Earthquakes. 120, 5,2000

Wang,X.,Wang,M.A.:Hyperchaos generated from Lorenz system,Physica A.387 (14), 37513758,2008

XuejunGao.:Bifurcation Behaviors of The two-state variable frictional law of a rock mass system, Int. J Bifur. Chaos.23,11,2013, DOI:10.1142/S0218127413501848 
Nonlin. Processes Geophys. Discuss., doi:10.5194/npg-2016-11, 2016

Manuscript under review for journal Nonlin. Processes Geophys.

Published: 1 February 2016

(c) Author(s) 2016. CC-BY 3.0 License.

YajingLiu.:Physical Basis of Aseismic Deformation Transients in SubductionZones,PhD thesis,

Harvard University,2007

Zhiren, N. and Dangmin, C.:Period-Doubling Bifurcation and Chaotic Phenomena in a Single Degree of Freedom Elastic System With A Two-State Variable Friction Law, in Nonlinear Dynamics and Predictability of Geophysical Phenomena (eds W. I. Newman, A. Gabrielov and D. L. Turcotte),

American Geophysical Union, Washington, D. C.. doi: 10.1029/GM083p0075,1994

http://in.mathworks.com/matlabcentral/fileexchange/233-let

8

It Jacobian matrix corresponding to the equilibrium point may be expressed as

\section{Appendix -I}

2

$$
\lambda^{4}+e^{\phi_{0}}\left(1+\rho+\rho_{1}+K-\beta_{1}-\rho \beta_{2}-\rho \beta_{3}\right) \lambda^{3}+
$$$$
e^{2 \phi_{0}}\left(K+\rho+\rho_{1}+\rho \rho_{1}+2 K \rho-\rho \beta_{1}-\rho \beta_{2}-\rho_{1} \beta_{1}-\rho \beta_{3}-\rho \rho_{1} \beta_{2}-\rho \rho_{1} \beta_{3}\right) \lambda^{2}
$$$$
+e^{3 \phi_{0}}\left(2 K \rho+\rho \rho_{1}+\rho \rho_{1} \beta_{1}-\rho \rho_{1} \beta_{2}-\rho \rho_{1} \beta_{3}+K \rho^{2}\right) \lambda+e^{4 \phi_{0}} K \rho^{2}=0
$$

$s_{0} \lambda^{4}+s_{1} \lambda^{3}+s_{2} \lambda^{2}+s_{1} \lambda+s_{4}=0$

where:

$\mathrm{s}_{0}=1$

$$
s_{1}=e^{\phi_{0}}\left(1+\rho+\rho_{1}+K-\beta_{1}-\rho \beta_{2}-\rho \beta_{3}\right)
$$$$
s_{2}=e^{2 \phi_{0}}\left(K+\rho+\rho_{1}+\rho \rho_{1}+2 K \rho-\rho \beta_{1}-\rho \beta_{2}-\rho_{1} \beta_{1}-\rho \beta_{3}-\rho \rho_{1} \beta_{2}-\rho \rho_{1} \beta_{3}\right)
$$$$
s_{3}=e^{3 \phi_{0}}\left(2 K \rho+\rho \rho_{1}+\rho \rho_{1} \beta_{1}-\rho \rho_{1} \beta_{2}-\rho \rho_{1} \beta_{3}+K \rho^{2}\right)
$$$$
s_{4}=e^{4 \phi_{0}} K \rho^{2}
$$

$$
\left.\begin{array}{ccc}
\rho e^{\phi_{0}}, & \left(1-\rho_{1}\right) e^{\phi_{0}},\left(\rho-\rho_{1}\right) e^{\phi_{0}} \\
0, & 0, & 0 \\
0, & -e^{\phi_{0}}, & 0 \\
0, & 0, & -\rho e^{\phi_{0}}
\end{array}\right]_{\left(\phi_{\mathrm{ss}}, \hat{\theta}_{\mathrm{sss}}, \hat{\theta}_{2 \mathrm{ss}}, f_{s s}\right)}
$$

eigen values in term of $\lambda$ is obtained from the

The Routh-Hurwitz criterion is used to get the condition for stability of the present friction model. The characteristic polynomial equation is obtained as $s_{0} \lambda^{4}+s_{1} \lambda^{3}+s_{2} \lambda^{2}+s_{3} \lambda+s_{4}=0$. After applying the Routh-Hurwitz criteria $s_{1} s_{2}-s_{0} s_{3}=0$ and $s_{1} s_{2} s_{3}-s_{0} s_{3}^{2}-s_{4} s_{1}^{2}=0$. These non-linear algebraic equations are in turn, solved numerically for evaluating critical stiffness. 